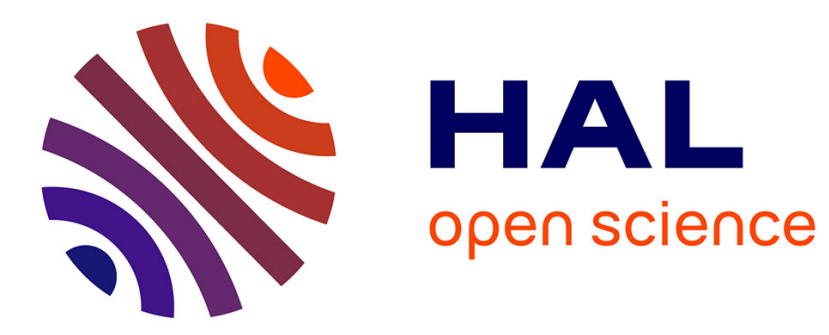

\title{
De la densité qui tue à la densité qui paye. La densité urbaine comme règle et médiateur entre politique et projet
}

Pierre Clément, Sabine Guth

\section{- To cite this version:}

Pierre Clément, Sabine Guth. De la densité qui tue à la densité qui paye. La densité urbaine comme règle et médiateur entre politique et projet. Les Annales de la Recherche Urbaine, 1995, 67, pp.72 83. 10.3406/aru.1995.1879 . hal-03240901

\section{HAL Id: hal-03240901 \\ https://hal.science/hal-03240901}

Submitted on 28 May 2021

HAL is a multi-disciplinary open access archive for the deposit and dissemination of scientific research documents, whether they are published or not. The documents may come from teaching and research institutions in France or abroad, or from public or private research centers.
L'archive ouverte pluridisciplinaire HAL, est destinée au dépôt et à la diffusion de documents scientifiques de niveau recherche, publiés ou non, émanant des établissements d'enseignement et de recherche français ou étrangers, des laboratoires publics ou privés. 


\section{De la densité qui tue à la densité qui paye. La densité urbaine} comme règle et médiateur entre politique et projet

Pierre Clément, Sabine Guth

\section{Citer ce document / Cite this document :}

Clément Pierre, Guth Sabine. De la densité qui tue à la densité qui paye. La densité urbaine comme règle et médiateur entre politique et projet. In: Les Annales de la recherche urbaine, №67, 1995. Densités et espacements. pp. 72-83;

doi : https://doi.org/10.3406/aru.1995.1879

https://www.persee.fr/doc/aru_0180-930x_1995_num_67_1_1879

Fichier pdf généré le 23/04/2018 


\section{Resumen}

De la densidad que mata a la densidad que paga

El urbanismo del siglo ha pasado de una crítica negativa del hacinamiento urbano a la elucidación de normas positivas de densidad. Desde el modelo de la ciudad-jardin al de la ciudad vertical, la gama de normas de densidad se diversifica. En los proyectos urbanos actuales, la fijación de coeficientes de uso del suelo es el teatro de tensiones políticas vinculadas a las estrategias financieras.

\section{Résumé}

L'urbanisme du siècle est passé d'une critique négative de l'entassement urbain à l'élucidation de normes positives de densité. Du modèle de la Cité-jardin à celui de la Ville Verticale, la gamme des standards de densité se diversifie. Dans les projets urbains d'aujourd'hui, la fixation des coefficients d'occupation du sol est l'enjeu de tensions politiques liées aux stratégies financières.

\section{Zusammenfassung}

Von der tödlichen zur lukrativen Bebauungsdichte

In unserem Jahrhundert ist der Städtebau von der Kritik am Zusammenpferchen der Einwohner zur Entwicklung positiver Normen für die Bebauungsdichte übergegangen. Zwischen dem Modell der Gartenstadt und dem der vertikalen Stadt erstreckt sich eine Skala von Dichtigkeitsnormen. Im heutigen Städtebau ist die Festlegung von Geschoßflächenziffern Objekt politischer Auseinandersetzungen und finanzieller Interessen.

\footnotetext{
Abstract

From density that kills to one that pays off

In this century, planning has gone from negative criticism of urban overcrowding to elucidating positive standards for density. From the Garden-city (Cité-jardin) model to that of the Vertical City, the range of density standards has diversified. In today's urban projects, determining the land use ratio is the stake in political tensions related to financial strategies.
} 


\title{
DE LA DENSITÉ QUI TUE Á LA DENSITE QUI PAYE
}

\author{
LA DENSITÉ URBAINE COMME RÈGLE ET MÉDIATEUR \\ ENTRE POLITIQUE ET PROJET
}

Pierre Clément, Sabine Guth'

A la fin du xixe siècle, ingénieurs, architectes, hygiénistes et politiciens utilisent un nouveau concept pour intervenir sur les villes : la densité urbaine. Apparaissant alors comme l'une des causes de l'insalubrité, des épidémies et de la mortalité des villes, cette notion de densité va alternativement et à travers l'évolution moderne de l'architecture et de l'urbanisme, servir d'indicateur, de preuve ou de justification aux politiques comme aux praticiens.

A l'origine moyen d'analyse critique, la densité va devenir outil, norme réglementaire fixant, pour chaque fonction urbaine, le coefficient maximum de construction autorisé sur un territoire donné. Le rapport direct entre mètres carrés constructibles et coût du foncier, donc rentabilité, va se substituer à celui entre homme et surface de territoire. C'est cette évolution que l'on se propose de mettre en relief au fil de quelques-unes de ses étapes, avec pour objectif de mieux comprendre comment la densité est outil ou contrainte au sein des processus de conception de la ville.

\section{Villes du XIXe siècle : nouvelle donne...}

L'industrialisation et notamment l'arrivée de nouveaux moyens de communication provoquent dans les villes occidentales du xIXe siècle d'importantes mutations urbaines en termes d'organisation, d'extension et de densification. Du croisement de diverses préoccupations liées à ce changement de nature naît un nouvel objet : l'habitat, comme enjeu d'une collaboration entre administration et spéculateurs, et auquel se confronte pour la première fois l'architecte.

Alors qu'émerge une nouvelle entité, l'agglomération, ce sont aussi bien les médecins, les ingénieurs ou les politiciens qui s'interrogent sur la ville et s'attachent à y corréler ordres spatiaux et modes de vie. Une partie de la critique qui s'élève alors s'exprime sous la forme de l'utopie urbaine et par le biais du modèle, et vise la société dans son ensemble.

\section{... projets utopiques et critiques}

R. Owen et son Village Industriel ${ }^{2}(1817)$, Ch. Fourier et son Phalanstère ${ }^{3}$ (1822), V. Considérant et sa Commune rurale $^{4}$ (1835) ou encore B.W. Richardson et son projet pour "Hygeia» ${ }^{5}$ (1876) constituent quelques jalons de ce courant à travers lequel se profilent les bases d'un urbanisme qui se veut science. Les organisations urbaines qu'ils imaginent, parfois véritables équations architectoniques, ont pour objectif d'optimiser le rapport entre territoire et population.

De petite taille et proches de la nature, les communautés urbaines qu'ils proposent ont une surface parfaitement délimitée et un nombre d'habitants fixé une fois pour toutes. Qu'elles soient basées sur la maison unifamiliale isolée ou l'habitation collective sous forme d'édifices unitaires, elles sont animées par un même souci de classement et de rationalisation. L'espace y est ouvert, aéré, planté et paysager, mais aussi découpé selon les fonctions et les activités, et mesuré avec précision. Il n'est jamais fait directement mention de la densité. Par contre, certains de ses indicateurs sont fréquemment exploités : depuis la quantité de population admise sur une aire urbanisée, la surface d'un lot destiné à recevoir une habitation ou encore les emprises bâties au sol. Décrivant par exemple la progression en densité du tissu - de plus en

Page 74 : figure 11. Hugh Ferris.

1. Le travail sur Paris a pu être mené grâce aux recherches de François Laisney sur le règlement. La recherche sur la ville américaine a été enireprise avec Sophie Clément-Charpentier, EHESS. Cet article est issu d'un travail exploratoire destiné à être développé par une recherche soutenue par le PIR-Villes, les rapports densité-projet, insuffisamment abordés ici.

2. CF. R. Owen, Textes choisis, introduction et notes de A. L. Morton, trad. française, Paris, éditions Sociales, 1963.

3. Cf. Ch. Fourier, Oeuvres complètes, Paris, éditions Anthropos, 1967 1968

4. Cf. V. Considérant, Destinée sociale, Paris, éditions Guy Durier, 1979.

5. Cf. B. W. Richardson, Hygeia, a city of health, Londres, Macmillan, 1876.

Les Annales de la Recherche Urhaine n' $07.0180-930-11.95 / 67 / 72: 12$ (C) METT 
«On doit tracer trois enceintes :

- la première contenant la cité ou ville centrale,

la deuxième contenant les faubourgs et grandes fabriques,

- la troisième contenant les avenues et la banlieue.

Chacune des trois enceintes adopte des dimensions différentes pour les constructions (...)

Toute maison de la cité doit avoir dans sa dépendance, en cours et jardins, au moins autant de terrain vacant qu'elle en occupe en surface de bâtiments.

L'espace vacant sera double dans la deuxième enceinte ou local des faubourgs, et triple dans la troisième enceinte nommée banlieue. Toutes les maisons doivent être isolées et former façade régulière sur tous les côtés, avec ornements gradués selon les trois enceintes, et sans admission de murs mitoyens nus.

Le moindre espace d'isolement entre deux édifices doit être au moins de 6 toises (...).

L'espace d'isolement doit être au moins égal à la demihauteur de la façade devant laquelle il est placé, soit sur les côtés, soit sur les derrières de la maison (...).

Sur la rue, les bâtiments, jusqu'à l'assise de la charpente ne pourront excéder en hauteur la largeur de la rue (...).

L'isolement sur les côtés sera au moins égal au 8e de la largeur de la façade sur rue (...) précaution nécessaire pour empêcher les amas de population sur un seul point (...).

Les places devront occuper au moins $1 / 8$ e de la surface.»

Fig. 1: Extrait de Ch. Fourier, Théorie de I'Unité universelle ou Traité de I'Association domestique agricole, Paris, 1822, cité par F. Choay dans L'urbanisme. utopies et réalités - Une Anthologie, éd. du Seuil, Paris, 1965. pp 97-98.

plus lâche - qui caractérise successivement les trois composantes (Cité, Faubourg, Banlieue) de sa Phalange pour 1500 personnes, Fourier en précise les rapports de proportion qu'il préconise entre quantités d'espaces libres et espaces bâtis, de même que les relations entre hauteurs bâties et espacements entre édifices et par rapport aux voies (fig. 1).

Ces propositions utopiques ne sont pas sans rappeler d'autres projets de cités idéales, également villes «finies», fondées parfois sur un même nombre d'habitants comme c'est le cas pour celles de Platon et de Léonard de Vinci, conçues pour 25 à 30000 personnes, chiffre que l'on retrouvera plus tard dans la Cité-jardin d'Ebenezer Howard. Mais, si les raisons des mesures et quantités de population choisies sont plus ou moins explicitées par les différents protagonistes, il n'existe aucune indication de la façon dont il est possible de maintenir stable le nombre d'habitants, et d'empêcher notamment la densification. C'est pourtant elle, et certaines de ses conséquences, qui est pour beaucoup à l'origine de la mauvaise image de la ville qui en fait, depuis le XVIIIle siècle, le véhicule de désordres et dégradations physiques et morales, et amène à la contestation de la Grande ville industrielle.

\section{L'émergence du concept de densité et la naissance d'un urbanisme scientifique}

Au cours du XIXe siècle, cette vision négative de la ville se voit confortée par les résultats de nombreuses enquêtes médicales ou démographiques qui mettent en évidence les concentrations de population toujours accrues et leurs conséquences. Pour évaluer et dénoncer ce phénomène d'entassement désigné par la pensée hygiéniste comme la cause principale des mauvaises conditions de vie et de l'insalubrité dont découlent épidémies et mortalité, on commence à utiliser la notion de densité urbaine. La première idée qui lui est associée est la mort. Cette problématique de la densité va se retrouver au cœur des préoccupations de l'urbanisme naissant qui, en exploitant le grand développement de l'outil statistique et devenant l'apanage de spécialistes en même temps qu'il se dépolitise, affirme progressivement sa tâche pratique et son caractère scientifique.

\section{Cerda à Barcelone : la densité indicateur d'analyse}

Alors qu'à Paris, dans le travail de grande ampleur que mène Haussmann accompagné de sociétés foncières et spéculateurs, s'esquissent les bases d'un urbanisme technique, en Espagne, Cerda fonde sa Théorie générale de l'urbanisation (1867) en mettant au point son plan pour Barcelone (1859). Mais quand Haussmann, à l'aide de Grandes Percées, d'un remaniement parcellaire et d'une nouvelle réglementation, se contente de réorganiser la ville existante, Cerda en propose une extension, de surcroît illimitée. La ville homogène et peu dense qu'il propose est en complète opposition avec la tradition de la ville dense enserrée dans son enceinte (Barcelone connaît alors une densité moyenne de 864 habitants à l'hectare ; à Paris, elle est de 714 hab/ha ${ }^{6}$ ). Son travail s'inscrit à la suite des troubles sociaux et des révoltes urbaines qui aboutissent en 1854 à la démolition des remparts de la ville, après avoir longtemps rencontré l'opposition des autorités militaires et des propriétaires fonciers de l'intérieur de la ville. L'expansion urbaine qui semble pouvoir résoudre tous les maux, hygiène, circulation et problèmes sociaux, est enfin possible.

Le dispositif qu'il conçoit pour l'Ensanche de Barcelone traduit son souci d'établir un système égalitaire susceptible d'empêcher la spéculation, facteur de densification et donc de mort, dont il décrit dans son ouvrage les processus et les formes (densification par superposition, mitoyenneté, réduction de la taille du logement...). Un plan en damier à base d'îlots carrés ou «manzanas», une

6. A. L. de Aberasturi, «Pour une lecture de Cerda», Introduction à I. Cerda La théorie générale de l'urbanisation, Paris, éd. du Sevil, 1979, p. 14. 
distribution uniforme des équipements et des parcs aux différentes échelles et une faible hiérarchisation du réseau viaire en constituent les principaux éléments. Formé par deux ou parfois trois barres isolées et alignées sur la rue, l'intérieur étant occupé par des jardins publics, chaque îlot s'ouvre à l'air et au soleil, conformément aux recommandations des hygiénistes de l'époque. Il en découle selon A. L. de Aberasturi une «densité moyenne réelle» de « 250 habitants par hectare ou, comme on disait à l'époque, $40 \mathrm{~m}^{2}$ par habitant, ce qui répondait aux standards recommandés par les comités d'hygiène publique» ${ }^{7}$ (fig. 2).

Si dans son plan Cerda n'énonce pas directement de chiffres de densité, celle-ci n'en est pas moins au centre de l'énorme travail d'analyse qui constitue selon lui la première phase de tout projet. Afin de «poser les problèmes en termes mathématiques" ${ }^{8}$, il fait appel à la statistique. La ville est instrumentalisée et devient objet d'étude. L'enquête que Cerda met au point s'intéresse à l'espace physique de la ville ou «contenant», à sa population ou «contenu», et surtout aux rapports entre eux appelés «fonctionnement» (fig. 3). Cerda s'intéresse à la quantité d'espace dont dispose chaque habitant : la densité est alors essentiellement un indicateur, un moyen d'analyse critique.

I. Du contenant : situation et circonstances topographiques et climatiques, membres, organisme, en plan et en élévation (...)

5A. 2B. C. Etat des maisons dans chaque îlot ou intervoies de la ville-matrice en 1859, avec l'indication des données suivantes: localisation, nombre, stratification, morcellement en appartements et expressions quantifiées de leur habitabilité selon le nombre d' individus qui s' y logent (...)

II. Du contenu : membres, organisme et loi de continuité de la population ; évolution historique de la population (...)

3A. 6C. Mortalité générale entre 1856 et 1865 selon l'étage habité, le parement de l'îlot correspondant, l'îlot, le quartier et le district (...)

III. Du fonctionnement, tel qu'il s'exprime dans les rapports entre le contenu et le contenant $(. .$.

2A. B. Superficie de trottoir, de demi-chaussée et intervoie correspondant à chaque habitant (...)

Conclusions

3A. 2B. D. (Etat du contenu par rapport au contenant) Par quartier, montrant que la mortalité varie en fonction de la classe sociale et que le maximum de décès est enregistré dans les îlots où prolifère la classe ouvrière alors que le minimum est atteint dans les îlots où il y a une forte proportion de riches bourgeois et que ceci correspond également à la densité de l'ilot."

Fig. 3: Extraits de la table des matières du Tome ll "Statistique urbaine de Barcelone" de l'édition originale de La théorie générale de l'urbanisation, I. Cerda, op. cit., pp. 215-222.

\section{Cités-jardins anglaises :} la densité standardisée

Théorisée en Angleterre par Ebenezer Howard dans Garden City of Tomorrow ${ }^{9}$ (1898), la cité-jardin se pose . comme alternative et lieu de résistance face au double phénomène de densification et d'extension démesurées de la ville - particulièrement aigu dans la périphérie londonienne qui connaît un afflux continu de populations rurales -, en proposant un ensemble de cités bien délimitées et au nombre d'habitants fixé par avance, séparées les unes des autres par des ceintures vertes.

Les formes urbaines mises en æuvre à Letchworth (construite en 1904 par R. Unwin et B. Parker sur le modèle économique de E. Howard), Hampstead Garden Suburb (réalisée entre 1905 et 1909 par R. Unwin et B. Parker) ou Welwyn (1919, combinant les théories d'Howard et les méthodes pratiques d'Unwin), à base de pavillons isolés, de "cottages» jumelés ou de maisons en bande regroupées en «close», et disposées en fonction de vues sur des parcs, sont relativement peu denses (fig. 4). Confortant les idées de communauté et de relation forte à la nature, elles répondent à l'énoncé d'un standard de densité maximum de 12 maisons par acre (soit 30 maisons par hectare) déduction faite des voies, pouvant toutefois varier en fonction de la nature et des dimensions des édifices. Ce qui est nouveau, c'est que Howard et Unwin, en plus de justifier le choix de cette valeur par le fait qu'elle permet d'assurer un bon éclairage naturel aux pièces en rez-de-chaussée au milieu de l'hiver, utilisent également un argument économique. Dans L'étude pratique des plans de ville"1) (1909), lorsque R. Unwin compare les coûts des voiries réglementairement nécessaires pour des densités de 20 puis de 12 maisons par acre (respectivement 50 et 30 maisons par hectare), il montre que dans le premier cas le prix élevé annule les gains au niveau de la valeur du sol par rapport au second exemple (fig. 5). La densité devient ainsi élément de programme : elle accompagne l'équilibre financier et fonctionnel que chaque cité-jardin vise à réaliser en redistribuant les ressources foncières à la collectivité.

Entre 1919 et 1939, l'Angleterre adopte des standards similaires, généralisant l'emploi de la maison «semidetached» et en bandes. Le modèle de la cité-jardin constituera jusqu'en 1959 la principale référence pour la construction de logements"'.

\section{A. L. de Aberasturi, op. cit., p. 31.}

8. La théorie générale de l'urbanisation, présentée et adaptée par A. L. de Aberasturi, p. 184.

9. Les cités-jardins de demain, Paris, Dunod, coll. Aspects de l'urbanisme, 1969.

10. Paris, Edition l'Equerre, 1984.

11. Cf. B. Goodchild «Housing layout, housing quality and residential densitys in Housing Review vol. 33, $n^{\circ} 4$, july-august 1984. 


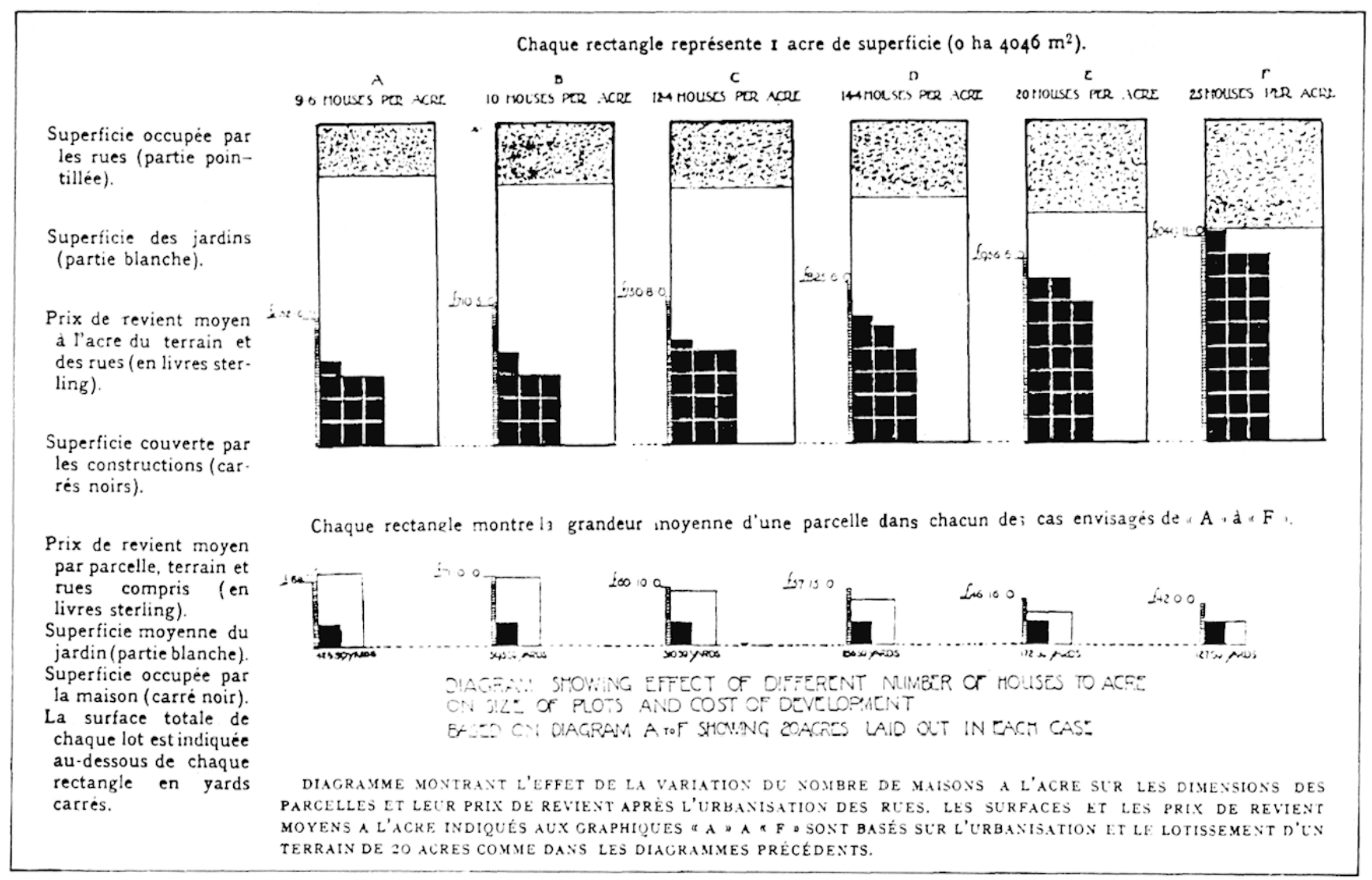

Fig. 5: "Dıagrammes III. -Grapnıque montrant ies conséquences de l augmentation au nombre de maisons ál acre sur la proportion de terrain à reserver aux rues et aux espaces bâtis, ainsi que sur les dimensions des parcelles à bâtir et sur le prix de revient: depuis 9,6 maisons àl'acre (24 maisons $a \dot{a}$ I'hectare), graphique A, jusqu'à 25 maisons àl'acre (61,8 maisons àl'hectare), graphique $F_{\text {"). }}$

R. Unwin, L'étude pratique des plans de villes, éd. I'Equerre, Paris, 1984.

\section{P. Abercrombie à Dublin : planification du logement et zoning}

Lorsqu'un concours pour concevoir le plan du Greater Dublin est lancé en 1914 par le Civics Institute of Ireland, plusieurs commissions d'enquêtes ont mis en évidence la présence dans la ville de nombreux taudis et quartiers congestionnés. Le projet ${ }^{12}$ que conçoit Patrick Abercrombie, lauréat du concours en 1916, conjugue relogement suburbain, développement d'un réseau de circulation et décentralisation industrielle, ce que complète une politique de provisions de parcs et de réserves foncières.

Posant avant tout le problème du logement en terme de densité, et afin de déterminer le nombre de personnes nécessitant d'être relogées, Abercrombie confronte entre eux résultats d'enquêtes et règlement du concours qui énoncent différents standards de densité acceptable en nombre d'habitants par unité de surface. Mais, plutôt que d'utiliser ces normes de densité globale, il en propose une autre, plus précise et donc plus proche de la réalité, appelée densité «nette» et calculée sur les surfaces résidentielles ou normal urban acre : hors grandes emprises - eau, chemin de fer, port, industries -, grandes institutions, parcs et autres grands terrains non occupés. De plus, Abercrombie ne pouvant distinguer précisément les surfaces liées aux commerces et bureaux fortement imbriquées aux résidentielles, il ajuste par souci de rigueur son standard à la hausse. Partant du nombre de 75 habitants maximum par acre «net» (soit $185 \mathrm{hab} / \mathrm{ha}$ ), il évalue à 59750 le nombre de personnes à reloger en zone extra-urbaine, avec l'argument du faible coût du terrain et donc de la possibilité de rentabiliser aménagements et constructions sans avoir recours à de la forte densité (fig. 6). Il établit alors un Plan de Zoning subdivisant la ville en différents secteurs selon les fonctions urbaines : Cité d'affaire au centre, zones industrielles en liaison avec le port, quartiers résidentiels séparés des précédents par des ceintures vertes et dotés de valeurs normatives de densité en nombre de logements par unité de surface (entre 16 et 44 logements par hectare selon les zones) (fig. 7).

Au fil du développement de l'urbanisme, l'usage de la densité se normalise en accompagnant le processus d'objectivation de la ville que traduisent des plans de zoning attribuant à chaque fonction urbaine un ou plusieurs secteurs dotés de coefficients de densité. Ce

12. CF. P. Abercrombie, S. et A. Kelly Dublin of the future - 1922, The University Press of Liverpool et Hodder \& Stoughton Lid. London, Publications of the Civics Institute of Ireland, 1922, $58 \mathrm{p}$. 

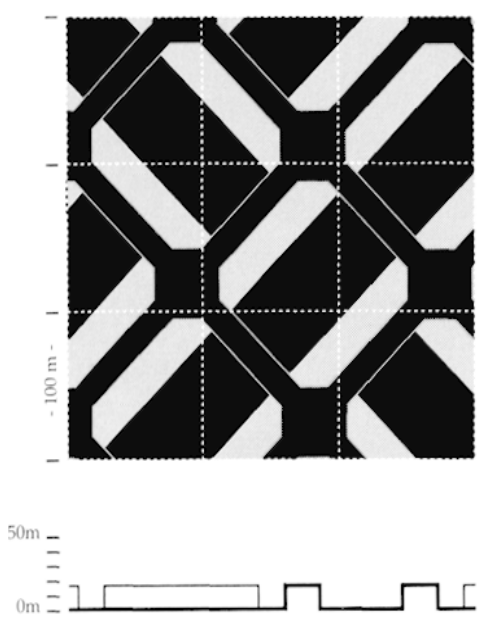

- alifot: emprise batte $0,32 \times 5$ niveaux $/ \cos 1,6$

Fig. 2 : Plan de Barcelone, 1. Cerda (extraits - ilots Cerda de $113 \times 113 \mathrm{~m}$ ). Note : Les plans et coupes des figures 2. $4,8,9,13$ et 14 sont présentés à la même échelle. sur une aire de 9 hectares : chaque carré en traits pointillés équivout à $100 \mathrm{~m} \times 100 \mathrm{~m}=1$ hectare. Les plans sont orientés Nord-Sud.

Les voiries et autres espaces libres publics délimitent l' "ilot" auxquels se réfc̀rent las données chiffrées. Celles-ci sont approximatives. Le chiffre donné pour le nombre de niveaux correspond aune moyenne.
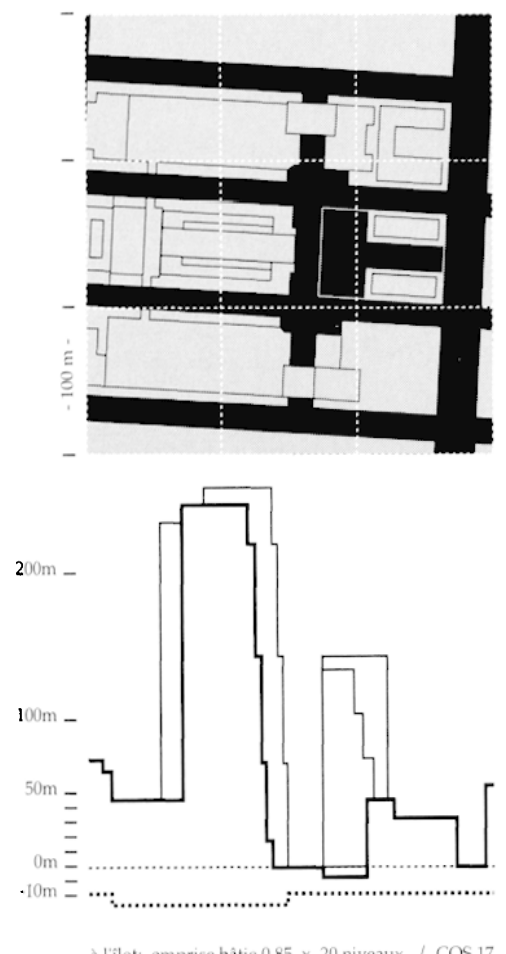

Fig. 10: Rockefeller Center (complexe de bureaux et commerces) ò Manhattan. New York; The Associated Architects Reinhard \& Hofmeister, Corbett. Harrison \& MacMurray, R. Hood, Godley \& Fouilhoux 1932-1940... (extraits).
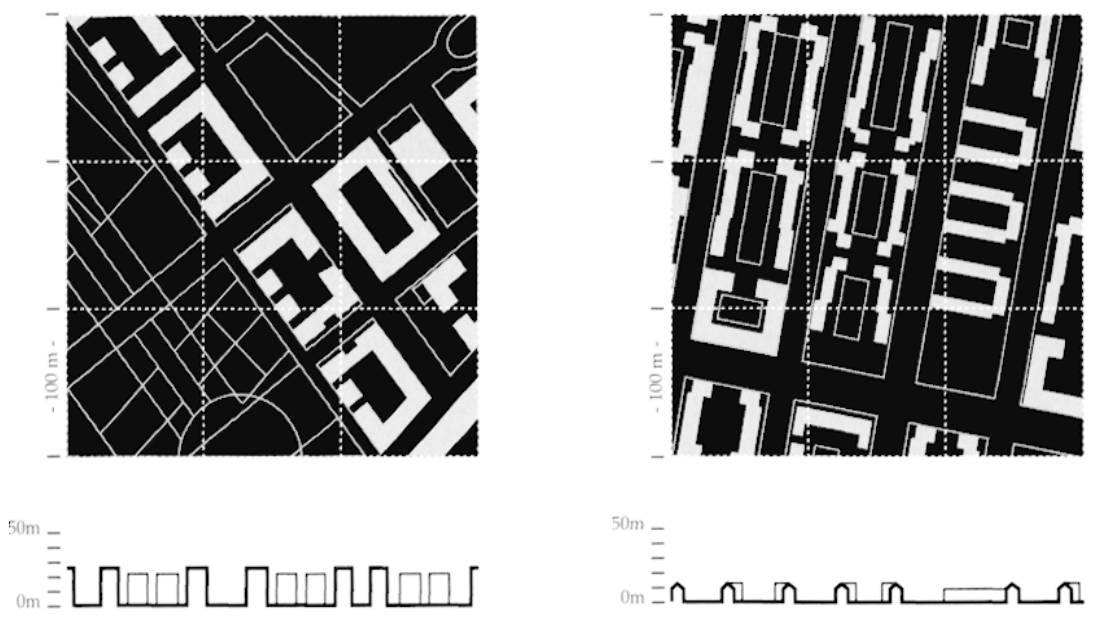

Fig. 4: Hampstead Garden Suburb, Londres, 8. Parker et R. Unwin (extraits).

Fig. 8: Sunnyside Gardens dansle Queens à New York. C. Stein etH. Wright, 1924... (extraits).

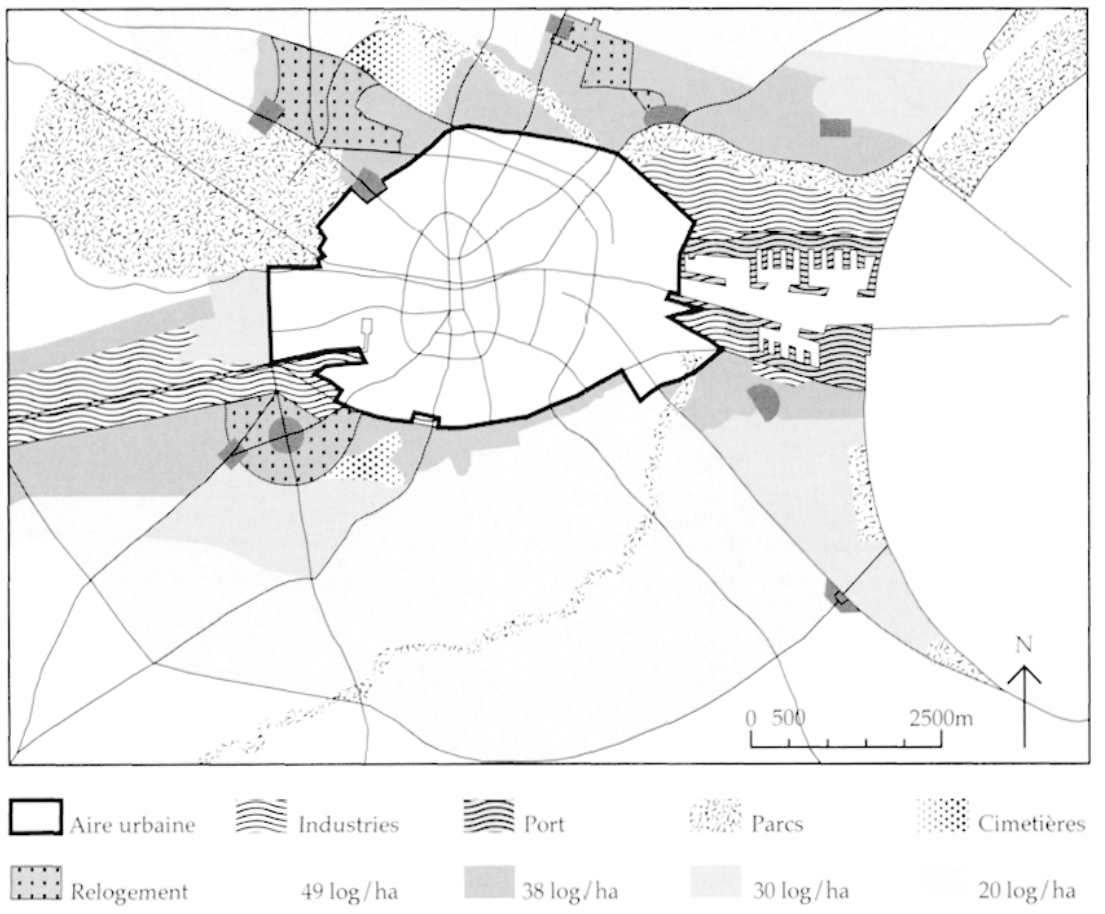

Fig. 7: Dublin, Plan de Zoning (1916) d'après P. Abercrombie, Dublin of the future, op. cit.
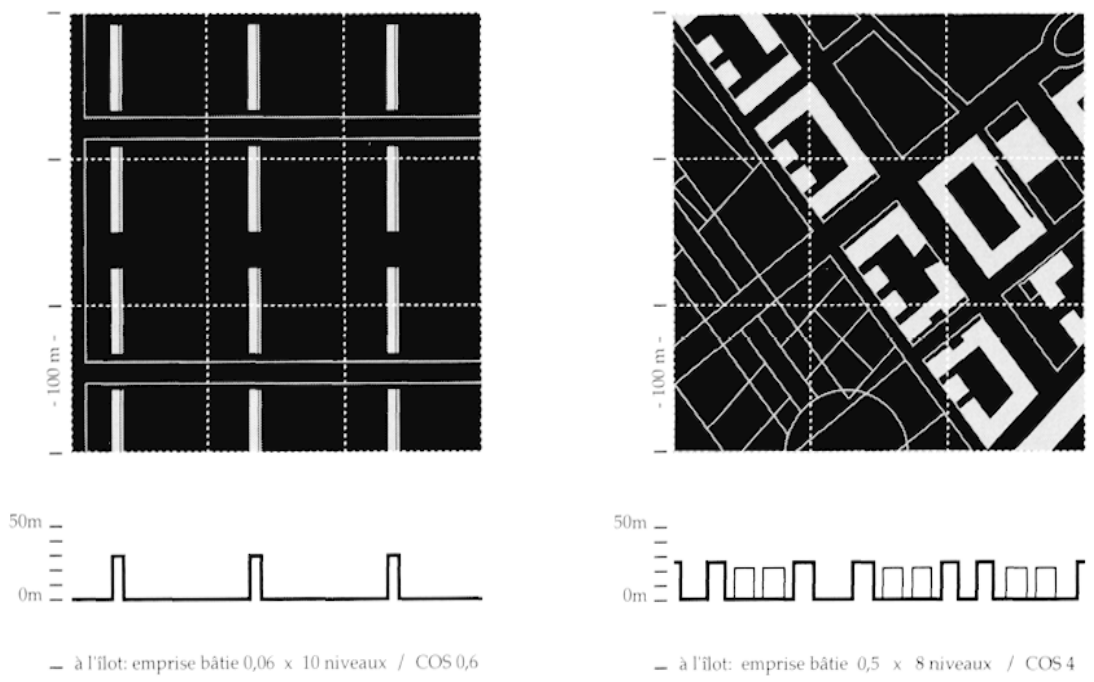

Fig. 13: Projet de Wohnhochhaus, W. Gropius, 1929-1930(Extrapolation).

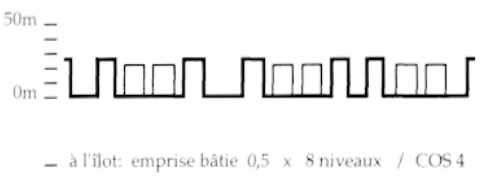

Fig. 14: ZAC de Bercy, Paris ; APUR, J.P. Buffi architecte-coordinateur (extraits). 


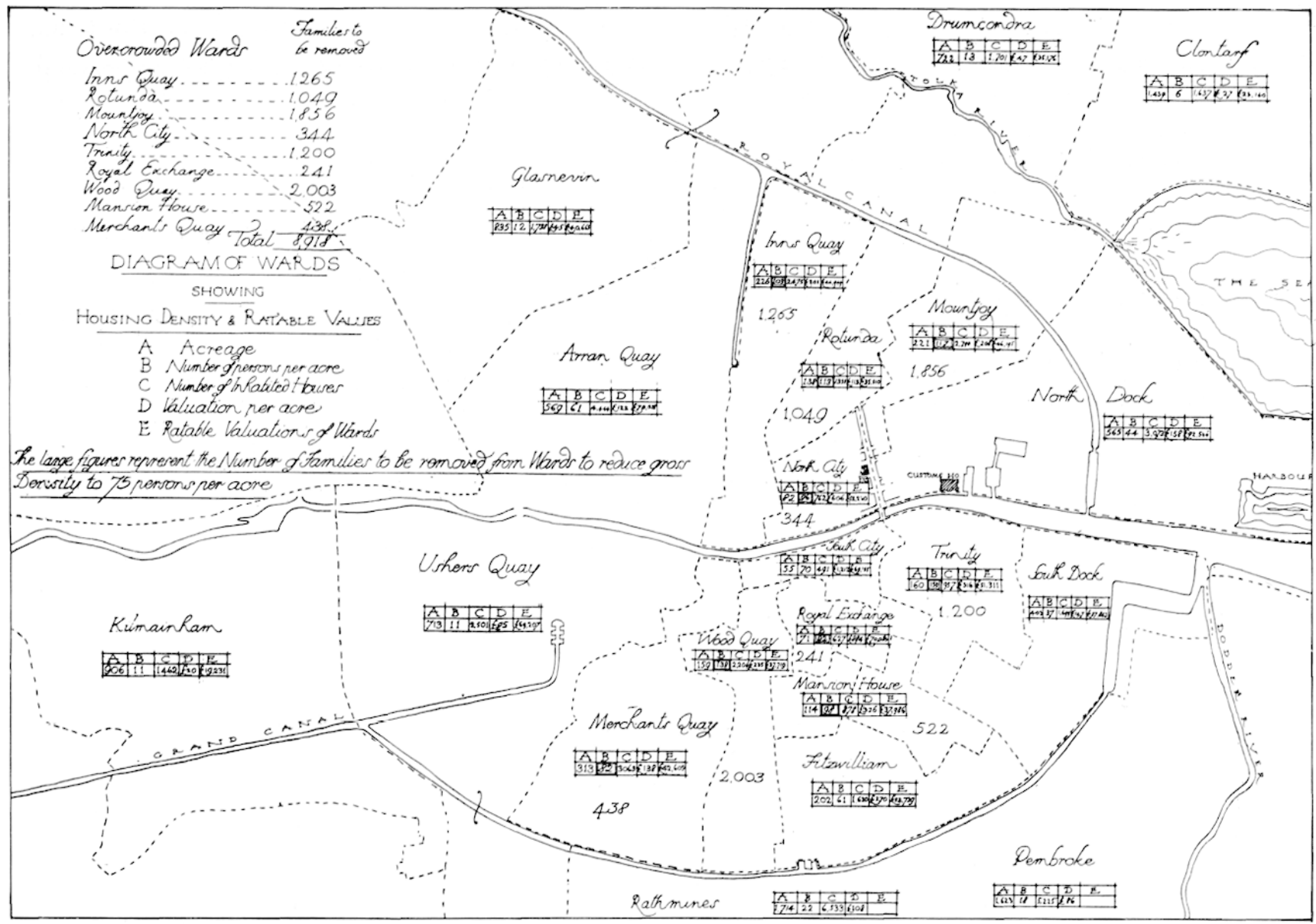

Fig. 6: Plan de Dublin. Relevé des superficies, valeurs foncières et densités par circonscriptions administratives (1916).

P. Abercrombie Dublin of the future, The University Press of Liverpool, Hodder \& Stoughton LTD. Londres, 1922.

A Superficie

BNombre d'habitants par acre ( 1 acre $=40,47$ ares)

CNombre de logements occupés

D Valeur foncière par acre

E Charge foncière par circonscription

zoning est un des éléments majeurs de la constitution de la ville américaine, objet de fascination et de rejet pour l'Europe.

\section{La ville américaine : zoning réglementaire...}

Face à l'urbanisation rapide qu'entraînent industrialisation et forte immigration, l'Amérique du XIXe siècle qui cherche à combiner valeurs familiales, religieuses et progrès scientifique et technologique, répond par une séparation des zones d'activités et des zones résidentielles à base de maisons individuelles proches de la nature qui incarne la morale ${ }^{13}$. Une opposition entre centre-ville, lieu de travail, et banlieue résidentielle intégrant la nature en s'étendant continuellement, se met progressivement en place, confortée par le développement d'une réglementation urbaine de type zoning qu'inaugure Los Angeles en 1908. La ville américaine qui en est issue est une agglomération fonctionnalisée, somme de quatre villes normalisées et fortement contrastées en matière de formes et de densités : CBD pour Central Business District, quartiers résidentiels, zones industrielles et secteurs commerciaux.

\section{... pour formes et densités extrêmes}

L'évolution des villes américaines est ponctuée de nombreuses vagues de migrations vers la banlieue. A base d'opérations de construction courantes ou spéculatives, de l'investissement de sociétés philanthropiques à profit limité ou de diverses politiques gouvernementales et municipales mises en place dans l'urgence de certaines situations comme après la première Guerre Mondiale, le développement périphérique qui en résulte est avant tout marqué par le mythe de la maison individuelle à la campagne. Il en découle une organisation spatiale extensive (fig. 8). Dans The disappearing city ${ }^{14}$ (1932) puis dans son projet pour Broadacre City ${ }^{15}$, F. L. Wright dévelop-

13. Cf. C. Ghorra-Gobin «Mythes fondateurs des villes américaines» in Etudes foncières $n^{\circ} 31$, juin 1986.

14. F. L. Wright The disappearing City, William Farquhar Payson, New York, 1932. Rev. ed. The Industrial Revolution Runs Away, New York, Horizon Press, 1969.

15. CF. P. Mantziaras Théories de la ville dispersée dans le XXe siècle, mémoire de DEA, sous la direction de Jean-Lovis Cohen et Hubert Damisch, Ecole d'architecture de Paris-Belleville, EHESS, 1994. 
pera de façon extrême la logique d'une ville américaine de très faible densité - dans certaines parties de son plan, chaque famille dispose d'un acre de terrain, soit 2,47 ha -, dispersée dans la nature, et rendue possible par le développement de l'auto : vision prémonitoire des suburbs illimités qui constituent la plus grande part de la ville américaine actuelle.

Dans le centre de cette même ville, l'autre part se construit au contraire sur de la forte densité et s'exprime essentiellement à travers la verticalité. L'invention de l'ascenseur (1859, Gaynor) permet d'augmenter considérablement les possibilités de hauteur d'édification. La limite liée à la distance maximum qu'une personne était censée pouvoir effectuer en montant des escaliers disparaissant, l'édifice devient la matérialisation directe de la valeur du sol (fig. 9). Une culture urbaine spécifique de l'hyper-densité se développe alors sur la base de tours, dont Manhattan en représente un exemple extrême. Au fur et à mesure des vagues d'immigration du XIXe siècle, la trame manhattanienne fait l'objet de spéculations et de constructions toujours plus grandes, donnant naissance à des gratte-ciel allant jusqu'à 60 étages. Cette densification massive que certaines mesures comme la «Nouvelle Loi» de 1901 entreprennent momentanément de freiner en limitant emprises au sol et hauteurs bâties, se poursuit dans les années de «laisser-faire» du début de siècle, engendrée par une pression économique sans pareille.

En 1916, la ville de New York tente de stabiliser la valeur foncière en adoptant la première réglementation des Etats-Unis combinant distribution des fonctions urbaines sur trois types de secteurs (résidentiel, commercial et industriel) et Zoning Envelop, un nouveau concept codifiant les dimensions et la forme des gratte-ciel en imposant cinq formules de recul par rapport à l'alignement sur la rue à partir de certaines hauteurs et en fonction de la largeur de voirie ${ }^{16}$. Mais les enveloppes définies sont généreuses et permettent toujours la mise en œuvre de fortes densités, ce qui contribue d'ailleurs au succès de leur principe (fig. 10). L'utilisation maximale des volumes réglementaires aboutit à l'émergence d'un nouveau type de gratte-ciel, dont des architectes et des peintres comme Hugh Ferriss ${ }^{17}$ exploitent l'esthétique métropolitaine (fig. 11).

Les fortes densités produites ne sont pas remises en cause lors de la révision du Zoning de 1961 qui introduit notamment la possibilité de rachat d'espace aérien voisin. Mais, en créant des Special Zoning District dans lesquelles est rendu possible et même favorisé le dépassement du nombre d'étages autorisés en échange d'un espace privé rendu public sous forme de plazza ou d'espaces verts, elle contribue à de grands changements dans la physionomie de la ville. Selon les préceptes du Mouvement Moderne, le bâti ne se réfère plus à la rue, mais il est déterminé par des ratios d'espaces libres et d'espacement entre édifices. La plupart des grandes villes des Etats-Unis vont s'inspirer de ce zoning de 1961 pour élaborer leur propre Zoning Code. La tour entourée d'espace ouvert (tower-in-open-space) devient le nouveau modèle de l'hyperdensité des centres-villes qui par ailleurs se vident peu à peu de leurs habitants.

\section{Mouvement moderne - Densité et rationalisation des formes}

A partir des années 20 , dénonçant le caractère anachronique et le manque d'efficacité de la ville héritée du XIXe siècle, les tenants du Mouvement Moderne proposent d'en rationaliser les formes en combinant zoning et standardisation. Cherchant à concilier densité rentable et acquis hygiénistes, leurs démarches aboutissent à une remise en question totale des formes architecturales et

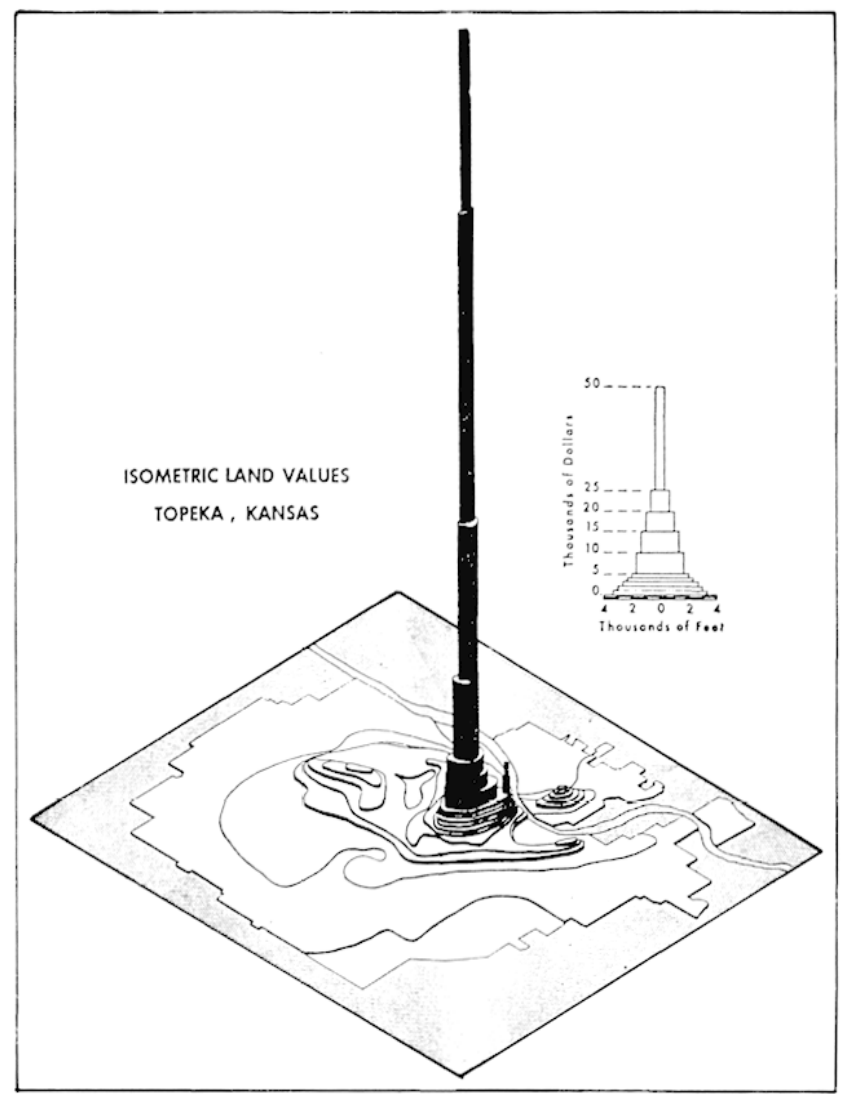

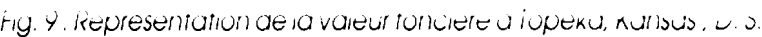
Knos Distribution of Land Values in Topeka, Kansas, Lawrence: Center for Research in Business, The University of Kansas, 1962.

urbaines. Avec l'abolition de la rue et une préférence pour la construction en hauteur, la notion de tissu urbain disparaît pour être remplacée par un système circulationunités bâties verticales dont l'emprise au sol est réduite au maximum, ce que compense mathématiquement le nombre de niveaux.

16. CF. Carol Willis «A 3-D CBD : how the 1916 Zoning Law shaped Manhattan's Central Business Districts", in Planning and zoning New York City - Yesterday, today and tomorrow, New Jersey, éd. Todd W. Bressi, 1993.

17. Hugh Ferriss The Metropolis of Tomorrow, 1929 ; rééd. Princeton Architectural Press, 1986. 
L'idée de la «ville dans un parc» sera partagée par les architectes fonctionnalistes de la mouvance des CIAM (Congrès international d'architecture moderne), et notamment par Le Corbusier dont on connaît le Plan Voisin de 1925 (emprise au sol de $5 \%$, densité de $3200 \mathrm{hab} / \mathrm{ha}$, soit cinq fois plus que les densités maximales du centre de Paris au XIXe siècle et dix fois les valeurs actuelles d'environ $250 \mathrm{hab} / \mathrm{ha}$ ) et son Unité d'Habitation de type barre qu'il présente en 1930 comme une alternative au pavillonnaire dont il dénonce le gaspillage de biens de consommation, de sol et de temps. Le principe est exploité très tôt dans le cadre de l'Allemagne industrielle où l'urbanisme est enseigné officiellement depuis la fondation du Bauhaus en 1919. Parallèlement à Marcel Breuer qui réalise en 1924 ses «logements bon marché» réunis dans de simples parallélépipèdes bâtis répartis dans la verdure, Ludwig Hilberseimer propose la même année sa "Ville verticale» puis, en 1927, sa «Métropole pour un million d'habitants» organisant, au sein de 120 îlots identiques surmontés d'édificesbarres, une séparation verticale entre lieux de travail et habitations ${ }^{18}$.

Walter Gropius, quant à lui, entreprend dès 1921 des recherches comparatives entre différentes formes d'habitation ${ }^{19}$. A travers une série de plans-masse, il fait varier à densité égale (400 lits par hectare) la hauteur et l'espacement entre barres parallèles disposées selon la direction Nord-Sud - «la plus favorable à une utilisation rentable du terrain $»^{20}$-, cherchant à démontrer rationnellement la supériorité des immeubles collectifs de grande hauteur. Alors que l'ensoleillement des logements et la densité sont simultanément augmentés par rapport à des constructions basses, l'emprise au sol est nettement inférieure : la nature peut ainsi pénétrer dans la ville. Selon Gropius, la forme optimum dans l'état du marché immobilier allemand d'alors est atteinte avec des immeubles de 10 à 12 niveaux, espacés selon une largeur égale à trois fois leur hauteur (soit $93 \mathrm{~m}$ pour 10 étages) (fig. 12, 13).

Dans leur recherche d'une architecture et d'un urbanisme objectifs, les fonctionnalistes du Mouvement Moderne allient à la notion de densité de nouvelles formes présentées comme la synthèse rationnelle et efficace entre espace, usage et économie, intégrant industrialisation et standardisation depuis l'échelle du logement jusqu'à celle du territoire. Les édifices «unités de grandeur conforme» sont disséminés au sein d'une «métropole totale» ordonnée par le zoning qui, en associant coefficients de densité, fonctions et localisation, apparaît comme l'instrument le plus efficace de la rationalisation recherchée. C'est sur la base de cette association densité-zoning qu'au cours du Xxe siècle se développe en France un urbanisme réglementaire, en même temps que s'inventent les mécanismes permettant de déjouer et transgresser les règles successives.

\section{La «cosification $»^{21}$ de la ville - Le cas de Paris}

Au cours du xxe siècle, la densité, qui ne faisait l'objet que d'un contrôle indirect par le biais de la réglementation de la forme, se voit attribuer un rôle direct dans la régulation de la ville occidentale. En France, elle n'apparaît sous une forme réglementaire qu'en 1959, à l'intérieur du Plan d'Urbanisme Directeur (PUD) de Paris. Jusque-là, les règlements successifs codifiaient un même système morphologique ${ }^{22}$.

A partir du XVIIe siècle, alors que la maîtrise de la croissance continue de la ville et la limitation de la densité de population étaient dévolues aux enceintes englobant progressivement les faubourgs, lieux de débordement, diverses règles de mitoyenneté, d'alignement, de profil et de hauteur du bâti sont mises en place. Exprimant des préoccupations de sécurité, de relation de voisinage et de rapport de l'espace privé à l'espace public, ces modes de contrôle de la forme ne manquent pas d'influencer la densité. Ils participent aux différents changements d'échelle comme aux phénomènes de monumentalisation de la ville sous le Second Empire, puis de verticalisation et d'individualisation de l'immeuble lorsque les préoccupations hygiénistes concernant l'aération et l'ensoleillement alimentent les nouvelles réglementations.

Entre 1959 et 1975, le PUD puis la loi d'orientation foncière de 1967 qui institue le POS (Plan d'Occupation des Sols) remettent totalement en question les instruments d'intervention conventionnels. Le CUS puis le COS (coefficients d'utilisation puis d'occupation du sol) qu'ils proposent fixent la quantité de surface de planchers constructibles par rapport à la surface du terrain, en fonction de la zone fonctionnelle dans laquelle ils se trouvent. Chaque secteur défini par le POS se voit ainsi doté d'un niveau de densité - COS moyens de $3(3,5$

\begin{abstract}
18. Cf. L. Hilberseimer, Groszstadtarchitektur, 1927 - Cf. A. de Poli «Les projets de la métropole des années 20 : références américaines", in J. L. Cohen et H. Damisch Américanisme et modernité, EHESS, Paris, Flammarion, 1993.

19. Walter Gropius, Construction horizontale, verticale ou de hauteur intermédiaire?, publié dans le recueil des CIAM, Verlag Englert und Schlosser, 1931 ; «Des logements au milieu d'espaces verts : un habitat d'avenir dans les grandes agglomérations urbaines», publié dans la revue Zentralblatt der Bauverwaltung, $n^{\circ} 49-50$ du 25 novembre 1931 ; textes présentés et annotés par Lionel Richard dans Walter Gropius, Architecture et sociêté, Paris, éditions du Linteau, 1995.
\end{abstract}

20. Walter Gropius, Architecture et société, op. cit., p. 101

21. On pourrait se laisser aller à interpréter le phénomène selon deux directions : cosification, à rapprocher de la chosification ou perte de la dimension humaine du rapport fondateur de la densité ; ou encore cosification, idée préétablie.

22. Cf. F. Laisney et R. Koltirine Règle et règlement. La question du règlement dans l'urbanisme parisien 1600-1902, rapport de recherche IPRAUS Ecole d'architecture Paris-Belleville, BRA, 1989.

Cf. F. Léonhardt Les règlements parisiens et le concept de densité, Séminaire Architecture Comparée, DEA Ecole d'Architecture Paris-Belleville, 1994. 
pour les zones d'activités à Paris) - qui fixe la valeur foncière du terrain et détermine son affectation en équipements publics. Ce passage de règles de délimitations formelles à des règles plus abstraites, plus qu'une volonté de renouvellement de la forme, traduit les nouvelles préoccupations réglementaires en matière d'économie, liées à une conjoncture immobilière propice. L'abandon de l'alignement et l'application indifférenciée de la règle de l'équivalence largeur de voie - hauteur de façade $(\mathrm{H}=\mathrm{L})$ favorisent l'édification en recul par rapport à la rue et de nombreux sauts d'échelle pouvant caractériser tout un secteur, à l'intérieur d'un périmètre opérationnel régi par un plan masse.

En 1975, un nouveau POS reflète l'analyse critique des nouveaux espaces urbains produits. Sans être annulées, les dispositions réglementaires précédentes sont adjointes de règles morphologiques : alignement bâti, gabarits indépendants de la largeur des voies et plafonnement des hauteurs de construction. L'ensemble favorise un îlot dense en périphérie et dégagé en son cœur, mais le principe du zonage est maintenu tout en étant adapté : 13 zones à vocations différentes sont chacune dotées de 3 COS d'habitat, de bureaux et d'activités, dont l'un des trois est favorisé en fonction de la destination prévue pour la zone (fig. 14).

La révision du POS de Paris adoptée en 1989 est marquée par une certaine complexification de la notion de densité avec l'introduction de la règle du COS de fait (qui permet de reconstruire une surface de plancher égale à la surface initiale existant sur le terrain, même si celleci dépasse le $\operatorname{COS}$ de la zone concernée) et la possibilité de dépassement du COS (système de marchandisation de la construction pour certains motifs d'architecture et d'urbanisme, soumis à autorisation de la Ville de Paris et en échange d'une taxe supplémentaire).

Cette dernière mesure ainsi que le relèvement général des COS bureaux provoquent de fortes réactions des habitants et associations, entraînant, dès 1994, une nouvelle révision du POS. Se préoccupant de mixité, celui-ci instaure une distinction entre activités et bureaux afin de contrecarrer les fraudes consistant à faire passer de l'un à l'autre les locaux correspondants. Les bureaux, dont les COS ont été abaissés, se développent alors essentiellement au sein des ZAC (Zones d'Aménagement Concertées). La règle du COS est également affinée en faisant apparaître systématiquement un COS incitatif de 4 pour les commerces et activités (sauf bureaux) en rez-dechaussée et au premier sous-sol, alors que les COS bureaux sont abaissés. L'organisation interne de la forme urbaine se trouve alors également régie par la réglementation de la densité. Des restrictions (imposées par le Conseil d'Etat) quant aux motifs architecturaux et urbains permettant d'obtenir un dépassement de COS sont également l'occasion de réguler plus fortement la forme urbaine, notamment en matière de comblement de «dents creuses».

Le POS, doté de son principal outil le COS, se voit donc
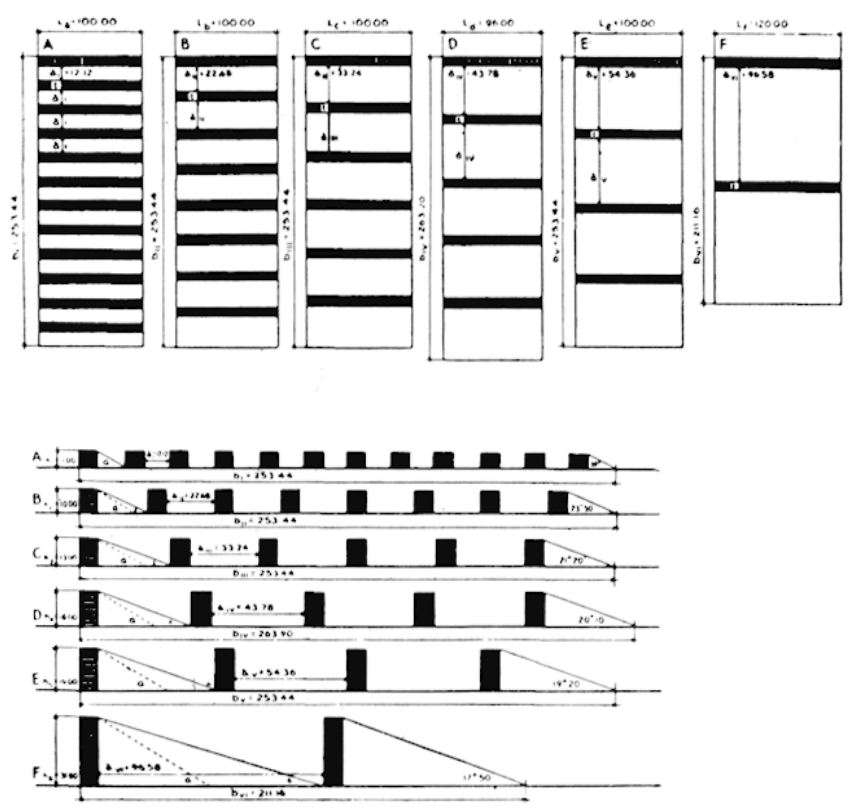

Fig. 12 : "Comparaison d'immeubles d'habitations de hauteurs différentes (2, 3, 4, 5, 6 et 10 étages) construits par alignement en rangées parallèles", W. Gropius, "Des logements au milieu d'espaces verts : un habitat d'avenir dans les grandes agglomérations urbaines", Zentralblatt der Bauverwaltung, $7^{\circ}$ 49-50, 25 nov. 1931, pp. 743-747 ; in W. Gropius, Architecture et société, éd. du Linteau, 1995, pp. 117-119.

progressivement attribuer un rôle plus large, tant dans l'uniformisation des profils que dans l'organisation interne de la forme urbaine. Traduisant une volonté de valorisation de l'économie, et alors que le nombre d'habitants sur une aire donnée n'en constitue plus la clé, cette utilisation de la densité sous forme de «cosification» de la ville n'est pas un phénomène isolé. Bien au contraire, elle s'inscrit dans une tendance qui se généralise aujourd'hui au niveau mondial. Mais l'accélération du renouvellement des règles depuis 30 ans est également révélatrice du fait que l'espace abstrait qu'elle suppose est vite rattrapé par la complexité des divers enjeux économiques et culturels qui caractérisent le tissu urbain. Les ajustements qui sont successivement mis en place, sans toutefois remettre en question les grands principes de base, tentent de parer aux effets induits et parfois pervers d'une réglementation au départ très libérale. Mais, dans l'ensemble, ils ne comportent aucun projet de ville.

\section{Densité et projet urbain}

Deux phénomènes liés à la notion de densité marquent aujourd'hui la ville occidentale. D'une part, un desserrement toujours plus important du tissu urbain, et d'autre part un émiettement de la ville par le développement de zones monofonctionnelles et homogènes.

Le choix du thème de la «tache d'huile» retenu pour la réunion annuelle des agences d'urbanisme françaises en 1992, est révélateur de l'intérêt croissant que suscite le premier phénomène. L'atlas publié à cette occasion montre que dans 22 agglomérations françaises, entre 


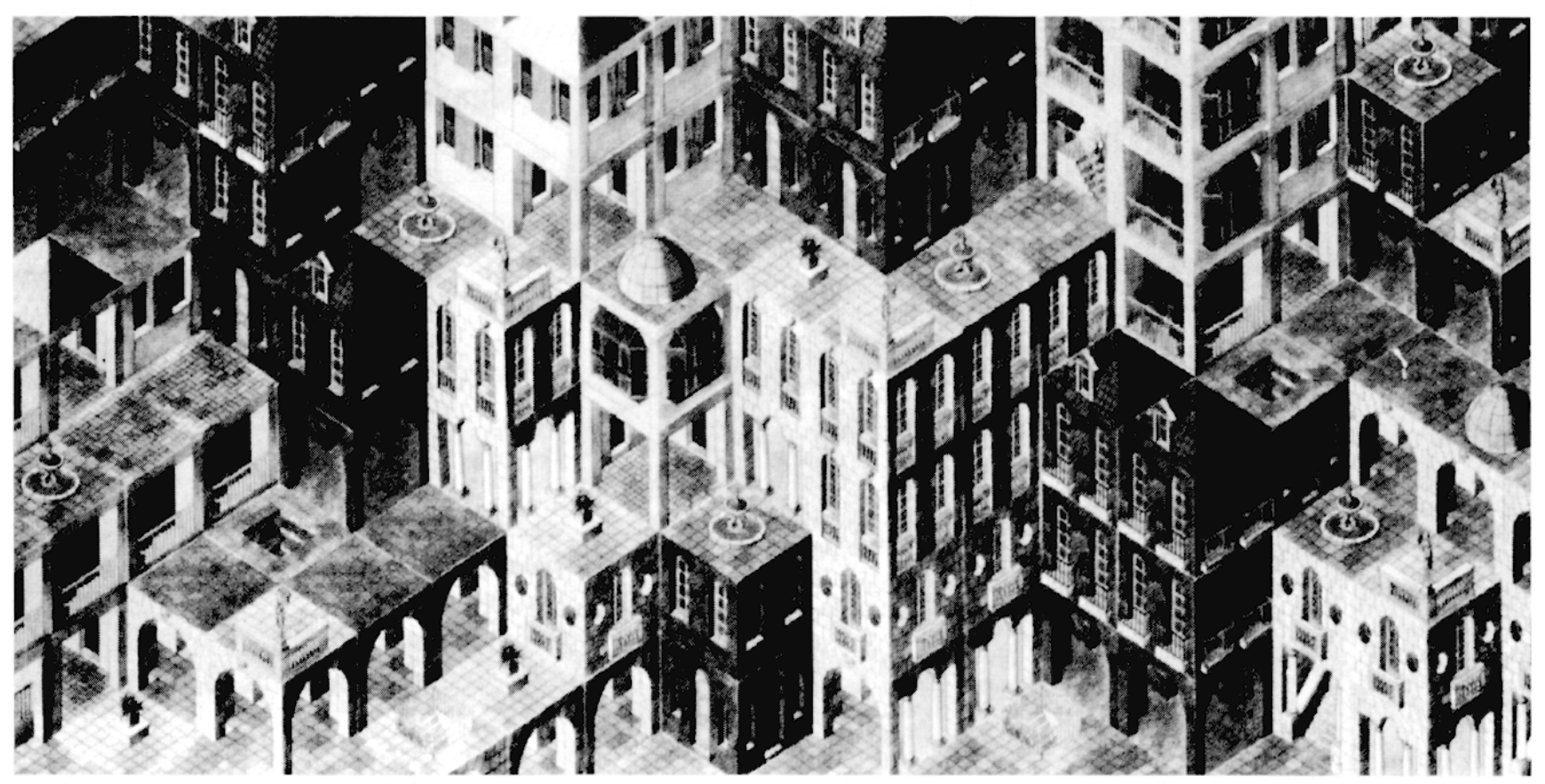

Fig. 15: Jean Pattou, Fresque "piranésienne" pour la station de métro, Euralille ; in AA n² 280, avril 1992, p. 132.

1950 et 1975 la population a doublé tandis que la surface occupée n'a augmenté que de 20 à $30 \%$, alors qu'entre 1975 et 1990, c'est la surface occupée qui a doublé pour une croissance de population de $25 \%$. A Paris, si le nombre d'habitants s'élevait en 1921 à 2900000 , en 1990, il n'est plus que de 2150000 . La dilatation de la ville pose de nombreux problèmes de coûts et de dysfonctionnements ${ }^{23}$ (gestion des espaces publics, transports, déplacements journaliers, etc.). Mais, face à la logique économique et financière, il devient urgent de poser également les problèmes en termes qualitatifs, et de réfléchir simultanément aux moyens de revivifier des centres désertés par la population et aux possibilités de concilier souci par rapport à l'environnement (consommation d'espace, pollution...) et qualité de vie pour les habitants, qui se traduit aujourd'hui essentiellement en termes de demande de logements individuels - et ce, même en villes nouvelles dont l'idée fondatrice était pourtant de fabriquer de l'urbain, de la ville dense.

En France comme dans d'autres pays, on commence à se pencher sur ces questions en tentant de cerner de plus près la notion de densité qui, par manque de rigueur et d'honnêteté, fait trop souvent l'objet d'utilisations opportunes et contradictoires. L'intérêt présent pour la densité s'exprime aussi bien sous la forme d'études de ses indicateurs, de sa perception ${ }^{24}$ ou des modes de densification, que dans la reprise de débats des années 70 sur les formes de «low rise - high density» combinant édifices modernes et organisations plus traditionnelles, dans des projets développant des formes permettant de «rendre acceptable» une certaine densité ou encore basés sur la notion de «sustainable communities» ${ }^{25}$, cherchant à concilier prise en compte d'un milieu naturel et vie sociale. Enfin, objet de représentations contrastées et d'esthétisation comme dans Delirious New York ${ }^{26}$ de R. Koolhaas, la densité devient discours. On se rappelle que la ville c'est la densité (fig. 15).

Le second phénomène, la fragmentation du territoire urbain en zones exclusivement vouées à une fonction et fortement séparées les unes des autres, est un effet direct du système de "cosification» auquel se superpose un ensemble de pratiques sectorielles particulièrement conséquentes en ce qui concerne les voies de circulation. La sérialisation programmatique et spatiale dont sont issus grands ensembles, centres commerciaux, mais aussi lotissements pavillonnaires, est source d'enclavement et d'exclusion, autant de problèmes exacerbés en situation de faible densité qui voit se renforcer l'éloignement et l'isolement de certains quartiers. En plus d'être la manifestation du zoning fonctionnel même souple de rigueur, ce compartimentage de la ville est lié aux mécanismes de nivellement et d'homogénéisation par l'utilisation maximale des possibilités de COS et de hauteursplafonds à l'intérieur de périmètres dont la délimitation très tranchée n'est pas toujours établie en corrélation avec le site. Dans le cas d'une ZAC, l'application d'une règle de densité sur l'ensemble de la zone et son développement comme une totalité achevée constituent souvent des facteurs aggravants de cette organisation ségréga-

23. Cf. V. Fouchier "Penser la densité» in Etudes foncières $n^{\circ} 64$, septembre 1994.

24. Cf. B. Goodchild, op. cit. : évoque des études montrant qu'il n'y a pas de relation directe entre densité résidentielle et satisfaction des habitants, celle-ci étant plus liée à la forme architecturale, l'environnement en général et la situation dans la ville.

25. Cf. S. Van der Ryn et P. Calthorpe Sustainable communities. A new synthesis for cities, suburbs and towns, San Francisco, Sierra Club Books, 1991. 
tive. Dans les secteurs sauvegardés qui composent une bonne part des centres-villes, ce sont avant tout des idées de protection et de reproduction de formes existantes que véhicule le POS. «(...) les procédures réglementaires actuelles, qu'elles soient d'ordre quantitatif : le COS, ou d'ordre esthétique : la notion de secteur homogène, ont pour effet de figer le tissu dans son état actuel, ou dans un état supposé idéal, d'empêcher son évolution normale et d'interdire l'exploitation des potentialités différentielles qui s'y sont peu à peu inscrites» ${ }^{27}$.

Se pose donc ici la problématique du projet comme démarche d'analyse et d'exploitation de ces potentialités par le biais de mécanismes et dispositifs spatiaux qui ne consisteraient pas uniquement à préserver des formes et des densités bâties qui, bien souvent, ne correspondent plus qu'à des enveloppes vidées de leur contenu (et ce, qu'il s'agisse d'immeubles, d'îlots ou même de quartiers entiers, notamment centres-villes). Si la ville, c'est la densité, il ne s'agit pas uniquement de densité bâtie, mais bien aussi d'événements et de significations. A l'échelle du projet d'architecture, le concepteur n'est pas directe- ment confronté au concept de densité, les choix le concernant étant établis en amont, aux niveaux réglementaire et de la maitrise d'ouvrage. Par contre, les questions qui se posent au concepteur urbain sont nombreuses, concernant aussi bien la délimitation d'aires d'affectation - au sujet desquelles on peut se demander s'il est systématiquement nécessaire d'affilier à une certaine surface fonctions et COS - que la définition de règles capables d'inscrire chaque projet dans ce rapport unité-diversité qui fait la ville. La densité, mesure globale de l'ensemble urbain et notion éminemment culturelle, est un réel support de planification. Elle n'en est pas le seul instrument.

\section{Pierre Clément} Sabine Guth

\footnotetext{
26. 1978, nouv. éd. 010 Publishers, Rotterdam, 1994.

27. Cf. D. Mangin et Ph. Panerai Le temps de la ville. L'économie raisonnée des tracés urbains, rapport de recherche LADRHAUS, Ecole d'architecture Versailles, 1988, p. 38.
}

Pierre Clément, architecte, est professeur à l'Ecole d'Architecture de Paris-Belleville et Directeur de l'IPRAUS (Institut Parisien de Recherche Architecture Urbanistique et Société), Laboratoire de l'Ecole d'Architecture de Paris-Belleville - Paris X Nanterre - Unité de recherche associée $n^{\circ} 1246$ du CNRS.

Sabine Guth, architecte, titulaire d'un DEA «Le projet architectural et urbain - Théories et dispositifs», est chargée de recherches à l'IPRAUS. 